\title{
Limited evidence that single-dose oral ibuprofen plus codeine is more effective for postoperative pain than either drug alone
}

\section{Jaime L Baratta, Kishor Gandhi, Eugene R Viscusi}

$10.1136 /$ eb-2013-101421

Jefferson Medical College of Thomas Jefferson University, Philadelphia, Pennsylvania, USA

Correspondence to: Dr Eugene R Viscusi

Department of Anesthesiology, Thomas Jefferson University, $111 \mathrm{~S} 11$ th Street, Suite G-8490, Philadelphia, PA 19107, USA; eugene.viscusi@jefferson.edu

Commentary on: Derry S, Karlin SM, Moore RA. Single dose oral ibuprofen plus codeine for acute postoperative pain in adults. Cochrane Database Syst Rev 2013;3:CD010107.

\section{Implications for practice and research}

- Professional societies (American Society of Anesthesiology (ASA) and the European Society of Anaesthesiology (ESA)) recommend maximising nonopioid over opioid analgesics and utilising a multimodal approach to perioperative pain management.

- This systematic review (SR) examines the effects of combining codeine and ibuprofen.

- The authors of SR conclude that ibuprofen and codeine combinations may provide enhanced efficacy.

- Future research should focus on whether combination agents provide better analgesia than their individual constituent alone.

\section{Context}

Postoperative pain control remains challenging. Historically, there has been a reliance on opioids for pain management. The American Society of Anesthesiology's (ASA's) most recent guidelines recommend maximising non-opioid agents with around-the-clock dosing and using opioids supplementally. ${ }^{1}$ Derry and colleagues performed a meticulous SR examining the analgesic efficacy of single-dose ibuprofen and codeine for moderate to severe postoperative pain. As with prior reviews by the group, ${ }^{2}$ this SR offers important insights into the potent nature of nonsteroidal anti-inflammatory drugs (NSAIDs) for the treatment of postoperative pain.

\section{Methods}

The authors reviewed six studies with 1342 participants undergoing procedures including episiotomy, caesarean section, other gynaecological surgery and surgical extraction of 1 to 4 impacted molars. Utilising electronic search engines, randomised, double-blind trials with at least 10 adult participants, comparing ibuprofen plus codeine to placebo or ibuprofen alone for the treatment of moderate-to-severe postoperative pain were identified. Two studies used medium-dose codeine $(<10 \mathrm{mg})$, the remainder used high dose $(>20 \mathrm{mg}$ ). Two independent review authors extracted and recorded the data and assessed for methodological quality. The outcome measures included: participants achieving at least 50\% pain relief over 4-6 h, median time and amount of rescue medication used and the occurrence of adverse events. Upon converting reported pain measures into participants with at least 50\% max total pain relief, relative risk (RR) and numbers needed to treat (NNT) to benefit were used to establish statistical difference.

\section{Findings}

The authors' findings demonstrated that the combination of ibuprofen plus high-dose codeine produced higher rates $(62 \%$ to $73 \%)$ of pain relief compared with the placebo (4\% to $38 \%$ ). Specifically in studies comparing $400 \mathrm{mg}$ ibuprofen plus codeine with placebo, the relative benefit of treatment was 4.1 and the NNT was 2.2. However, when comparing $400 \mathrm{mg}$ ibuprofen plus codeine to the same dose of ibuprofen alone, the relative benefit was 1.4 and the NNT was 7.1, demonstrating that the combination was more effective than ibuprofen alone, however, the statistical significance was just reached. The authors found that $28 \%$ of patients experienced adverse events in the high dose codeine group compared to $18 \%$ with the placebo $(\mathrm{RR}=1.2)$.

\section{Commentary}

SRs of acute pain trials can be cumbersome and challenging. Although a meta-analysis can provide statistical power and the ability to extrapolate to the general population, the inherent weakness is the heterogeneity of study populations. Acute pain trials are notorious for introducing bias from small treatment groups, high rates of placebo efficacy and low rates of success with active medications. The authors of this review minimised such biases with the use of random sequence generation, allocation concealment and blinding of outcome assessment by independent reviewers. However, this review has the inherent weaknesses posed by a meta-analysis with limited studies measuring adequate treatment groups. The consequence may be the generation of an inaccurate conclusion.

We must consider the emerging concepts of multimodal analgesia: that one or more non-opioids should be employed as around-the-clock agents, shifting opioids to a role as rescue agents. The concern for opioid related adverse events as well as misuse/abuse may challenge traditional reliance on opioid analgesics and opioid/non-opioid combinations with fixed-dose ratios. A prior SR strongly supports the analgesic efficacy of non-opioids when compared to opioids and combination agents. $^{2}$ Codeine presents unique 
challenges because a segment of the population does not have the P450 2D6 pathway to metabolise codeine to morphine, rendering it inactive.

The analysis of $400 \mathrm{mg}$ ibuprofen and high dose codeine versus placebo showed statistical difference. It is unclear if the superiority is from NSAIDs or codeine alone. When examining ibuprofen and codeine (all doses) versus ibuprofen alone, the statistical significance was barely reached (risk ratio $1.26,1.01$ to 1.57 ). This review is unable to ascertain the efficacy of combination drugs compared to NSAIDs alone. Despite its limitations, this review demonstrates some benefit of combination drugs in the treatment of acute pain. This SR will further the discussion on the potency of NSAIDs compared to opioids in the treatment of acute pain.
Competing interests ERV's institution has received research grants from AcelRx, Adolor, Cadence, Cumberland and Pacira. ERV also acts as a consultant for AcelRx, Cadence, Cubist, Incline/Medicines Company, Pacira and Salix.

\section{References}

1. ASA Task Force on Acute Pain Management. Anesthesiology 2012;116:248-73.

2. Moore RA, Derry S, McQuay HJ, et al. Single dose oral analgesics for acute postoperative pain in adults. Cochrane Database Syst Rev 2011;(9):CD008659. 